\title{
Les enjeux de la nouvelle directive-cadre sur les ressources en eaux souterraines
}

\author{
The new framework directive stakes for groundwater resources
}

par B. Kaczmarek

Délégation de Bruxelles - Agences de l'Eau

The future water framework directive aims to reach a good quality for surface water and groundwater resources all over European Union territory within two decades. The chosen approach-implementation of detailed action programs within hydrographic basins consisting of regulatory and economic measures will deeply modify national water policies in the 15 member-States of the European Union, by setting up precise methodologies. A special attention has been given to groundwater resources protection in this directive. This specific area related to groundwater will be presented within the new general framework created by the directive.

\section{I — L'UNION EUROPÉENNE ET LA PRO- TECTION DES RESSOURCES EN EAU}

La politique de lutte contre la pollution de l'eau est la plus ancienne et la plus complète au sein de l'action environnementale de la Communauté. Depuis 1975, plus de 30 directives ou décisions ont été adoptées couvrant à la fois la pollution de l'eau douce et l'eau de mer.

Cette politique se présente en grande partie selon une double approche, la première étant de nature générale et consistant en une lutte contre le déversement de substances dangereuses dans l'environnement aquatique de la Communauté, la seconde étant d'une nature plus spécifique et visant à définir certaines normes de qualité sur des zones particulières où des usages particuliers doivent être protégés.

Diverses directives ont été ainsi adoptées, principalement au cours de la période $1975-1980$ et on peut citer les principales d'entre elles : la directive sur les rejets de substances dangereuses de 1976 a permis de développer des réglementations sur la limitation de rejets d'une trentaine de substances considérées comme dangereuses. Les directives relatives à des usages particuliers telles que la production d'eau potable (1975), la pêche (1978), la conchyliculture (1978), la baignade (1976) ont permis d'élargir notablement le champ d'action de la politique communautaire. La directive sur l'eau potable de 1980 actualisée en 1998 établit des normes de qualité identiques (et d'ailleurs très élevées) pour tous les Etats de l'Union.

Des directives importantes ont été adoptées plus récemment. La directive sur l'épuration des eaux résiduaires urbaines de 1991 sert de cadre à la définition de programmes d'assainissement pour l'ensemble des agglomérations urbaines de l'Union. Elle est donc la cause première de l'important effort financier développé dans ce domaine au cours des dix dernières années. La directive sur la prévention des pollutions par les nitrates d'origine agricole a, elle aussi, été adoptée en 1991. Elle pose les principes d'une politique de prévention des pollutions diffuses pour le secteur agricole dont les effets se développeront dans les prochaines années.

Au cours de la période qui a suivi l'adoption des deux directives de 1991, il est apparu aux différents responsables nationaux des politiques de l'eau que l'importance des engagements financiers nécessités par l'application de la réglementation conduisait à s'interroger sur son efficacité et en particulier sur la bonne harmonisation des différentes directives. C'est ainsi qu'en 1995, le Conseil et le Parlement Européen ont souhaité un réexamen approfondi de la politique communautaire de l'eau. Ils ont alors demandé à la Commission d'élaborer une proposition de directive cadre. Une telle proposition fut présentée début 1997 et elle fit l'objet de débats approfondis au sein du Conseil et du Parlement. Au terme de la procédure dite de codécision, le texte définitif devrait être adopté dans les toutes prochaines semaines et l'on peut d'ores et déjà présenter les lignes générales de cette directive qui guideront la politique communautaire de l'eau des prochaines décennies.

\section{II —L'OBJECTIF PRINCIPAL DE LA DIRECTIVE CADRE}

Une directive cadre idéale devrait se proposer de répondre aux quatre objectifs d'une politique durable dans le domaine de l'eau que sont: l'approvisionnement en eau potable des 
populations, l'approvisionnement en eau à d'autres usages marchands, la protection de l'environnement, la réduction des conséquences des inondations et sécheresses. Ces objectifs peuvent cependant s'avérer contradictoires les uns envers les autres et il est donc nécessaire de mettre en place une hiérarchie. La protection de l'environnement a été considérée par la Commission (et elle a été suivie sur ce point par le Conseil et le Parlement) comme l'objectif principal qui permet d'ailleurs de répondre à la réalisation des deux premiers objectifs en assurant le maintien de ressources en eau brute de bonne qualité. Le quatrième objectif n'a pas été retenu comme devant être directement l'objet de cette directive compte tenu, notamment, du partage de compétences existant entre le niveau communautaire et les niveaux nationaux ou locaux : la directive pourra cependant, dans la mesure du possible, contribuer de manière indirecte à sa réalisation.

\section{I LA STRUCTURE DE LA DIRECTIVE CADRE}

La directive cadre a été construite selon un fil conducteur définissant les objectifs à atteindre à partir d'un état des lieux à établir. Elle précise les instruments dont il convient de se munir (méthodes, surveillance) afin d'établir une programmation des moyens intégrée dans une démarche de planification. Enfin, apparaissent des objectifs annexes ou des dispositions à caractère administratif permettant de compléter le dispositif.

\subsection{Les objectifs}

L'objectif global de la directive énoncé à l'article $1^{\text {er }}$ est d'atteindre un bon état des eaux. Pour cela, il convient de prévenir toute dégradation et d'améliorer la qualité de cellesci. Il faut également promouvoir une utilisation durable de cette eau en organisant les éléments d'une protection à long terme des ressources disponibles. Accessoirement, il est nécessaire de contribuer à l'atténuation des effets des inondations et sécheresses. Sur demande du Parlement, deux volets supplémentaires seront ajoutés : l'un concerne la réduction et cessation des rejets de substances dangereuses et l'autre, la diminution spécifique de la pollution anthropogénique des eaux souterraines.

Afin de déterminer le cadre juridique prévu dans cette directive, l'article 2 présente un ensemble de définitions permettant de préciser ce que seront les obligations créées. Outre les diverses définitions relatives à la qualité des différentes masses d'eau - la masse d'eau étant l'entité homogène dont on suivra la qualité - apparaissent les définitions du bassin hydrographique et du district hydrographique.

L'article 3 définit le cadre spatial dans lequel l'action devra être conduite. La création d'une entité administrative le district hydrographique - dont le territoire correspond à un groupement de bassins hydrographiques est l'élément central de cette désignation. Une ou des autorités compétentes devront être chargées d'appliquer un certain nombre de règles prévues aux articles ultérieurs et en particulier la mise au point et l'application d'une planification détaillée. Des dispositions spécifiques quant au rattachement des nappes souterraines et à la manière de traiter les bassins internationaux y sont précisées. Un certain nombre de dispositions à caractère administratif et notamment le délai maximum prévu pour la création des districts (en principe trois ans) sont précisées.

L'article 4 précise les conditions de réalisation de l'objectif général défini à l'article ler. En particulier, il est prévu un délai de réalisation ainsi que les modalités particulières s'appliquant aux divers types de ressources aquatiques concernées (eaux de surface, eaux souterraines, canaux et systèmes fortement modifiés par l'homme, ...). Deux types de dérogations pour l'atteinte des objectifs dans un temps limité sont prévus : d'abord, un report d'échéances dû à des raisons techniques et économiques peut être accordé. En second lieu, l'objectif lui-même peut être rendu moins strict lorsqu'il est estimé - par les Etats - que la ressource concernée ne peut raisonnablement pas être l'objet d'un objectif aussi ambitieux.

Par ailleurs, des modalités annexes permettent de tenir compte des périodes d'inondation et de sécheresse ainsi que de préciser les obligations des Etats-membres. L'ensemble des dispositions de cet article 4 ont fait et continuent de faire l'objet d'âpres discussions entre le Conseil et le Parlement, ce dernier souhaitant le délai le plus court possible et le minimum de dérogations. Pour l'instant, on peut penser que l'obtention de l'objectif général de bonne qualité est programmé d'ici une quinzaine d'années avec de larges dérogations prévues pour une douzaine d'années supplémentaires. Pour ce qui est des dérogations relatives à l'obtention de l'objectif de bonne qualité, la discussion se concentre autour de dispositions qui, d'une part, n'interdiraient pas le "développement humain durable » et qui d'autre part, n'entraîneraient pas de «coûts disproportionnés".

\subsection{L'état des lieux}

Après avoir défini l'objectif dans les premiers articles, la directive s'attache à préciser une méthodologie de planification et d'actions dans les articles suivants. Le premier volet de cette méthodologie consiste à préparer une analyse globale du district et l'article 5 présente les éléments détaillés d'une telle étude : aspects géographiques, démographiques, économiques et techniques. L'article 6 permet d'adjoindre à cette analyse l'ensemble des caractéristiques environnementales particulières du district sous la forme d'un registre des zones protégées. Enfin, l'article 7 précise les conditions de captage des eaux du district pour répondre à l'usage prioritaire défini à l'article ler qui est celui de l'alimentation en eau potable des populations. Au total, et de fait, ces trois articles définissent un état des lieux des ressources en eau du district dans leurs rapports avec la population et l'économie.

\section{- 3.3 Les instruments nécessaires}

Après avoir défini cet état des lieux, la directive précise qu'il sera fait appel aux trois instruments particuliers que sont pour l'aspect technique, les programmes de surveillance de l'état des systèmes aquatiques, pour le domaine économique, le biais de la tarification de l'eau et enfin pour l'aspect réglementaire, il est fait appel à l'approche combinée.

L'article 8 précise les conditions générales de surveillance de l'état du milieu naturel et il renvoie à une annexe (l'annexe $\mathrm{V}$ ) pour définir très méticuleusement la manière de suivre l'évolution de ce milieu naturel dans toutes ses composantes.

L'article 9 met en place les lignes directrices d'une tarification incitative devant permettre d'apporter une contribution à l'objectif général d'une bonne qualité du milieu naturel. De par les conséquences économiques, politiques et sociales que ces modalités peuvent instaurer (notamment en matière de tarification de l'eau à usage agricole), cet article a fait, lui aussi, l'objet de débats approfondis entre le Parlement et le Conseil. Il semble aujourd'hui acquis que le concept de la couverture totale des coûts par secteur (qui était le projet initial de la Commission) sera largement amendé et que les Etats-membres auront toute latitude pour utiliser ce concept comme ils le souhaitent.

Enfin, l'article 10 précise ce qu'est «l'approche combinée " ; il indique, en particulier qu'il convient d'abord de mettre en place des contrôles d'émission basés sur les meilleures techniques disponibles (MTD), ainsi que de définir des valeurs limites d'émission pertinentes et de mettre en 
œuvre les meilleures pratiques environnementales pour les pollutions diffuses. S'il s'avère alors que l'état du milieu naturel n'est pas encore de qualité suffisante, il est alors nécessaire de rendre plus stricts encore les contrôles d'émission. Des discussions se poursuivent sur la prise en compte obligatoire ou non des petits rejets - et la définition de ces petits rejets - dans les mécanismes d'application de l'approche combinée.

\section{- 3.4 La programmation}

Les mesures détaillées nécessaires dans chaque district hydrographique afin de répondre à l'objectif général de la directive sont précisées à l'article 11 qui traite du "programme de mesures». Ces mesures sont de deux ordres : d'abord, des mesures dites de base qui sont notamment des mesures à caractère réglementaire et présentent, par exemple, les caractéristiques permises pour chaque rejet polluant, caractéristiques bien entendu conformes aux exigences communautaires et à l'approche combinée définie à l'article 10. Ces mesures de base peuvent être aussi relatives à la tarification incitative dans la mesure où elles suivent les prescriptions prévues à l'article 9. Ces mesures de base incluent enfin le contrôle des captages d'eau. Des mesures dites complémentaires peuvent faire partie du programme. Ces types de mesures sont spécifiées de manière indicative dans une annexe (l'annexe VI) et elles peuvent, par exemple, inclure des accords volontaires, des codes de bonnes pratiques, des mesures particulières de recyclage, des projets de recherche, d'éducation, des instruments économiques ou fiscaux,... Le texte définitif inclura des dispositions plus précises sur tous les rejets diffus tout en laissant aux Etats membres une large latitude de rechercher les meilleures solutions possibles.

\subsection{La planification}

Afin de mettre le programme de mesures en perspective et de le rendre légitime aux yeux des usagers, il est indispensable d'achever le dispositif en mettant en œuvre une démarche de planification. C'est tout l'objet des articles 13, 14 et 15 que d'exposer ce processus de planification.

L'article 13 définit les plans de gestion du district hydrographique et renvoie dans une annexe (l'annexe VII) aux détails de ces plans. D'une manière générale, ces plans reprennent l'ensemble des volets précédemment mis au point : l'analyse du district, le registre des zones protégées, le programme de mesures. L'article 14 insiste sur la nécessaire participation du public à la démarche de planification et prévoit les procédures à suivre afin d'assurer cette participation. L'article 15 précise les conditions de "rapportage " aux instances communautaires et plus particulièrement à la Commission.

\subsection{Les stratégies}

Les principales dispositions d'un cadre général pour la politique communautaire de l'eau ayant été reprises dans ces articles 1 à 15 , il a semblé utile d'ajouter un volet particulier qui relève de la réduction et de l'élimination des substances dangereuses. En effet, constat a été tiré d'une trop faible efficacité de la directive 76/464/CEE qui est l'instrument central de cette politique et qui n'a pas véritablement permis de lutter contre ce type de pollution.

L'article 16 prévoit donc une évolution notable de la méthode suivie jusqu'à présent en préconisant des «stratégies " autorisant le recours à des procédures simplifiées d'évaluation des risques d'écotoxicité et de toxicité. Une première liste d'une trentaine de substances dites prioritaires vient d'être publiée et l'on peut penser que ces substances feront l'objet de mesures très restrictives d'utilisation dans un proche avenir. D'importantes discussions se poursuivent quant au devenir de ces substances dangereuses - le Conseil proposant d'introduire une notion supplémentaire de substance prioritaire présentant un risque inacceptable...

Enfin, le corps de cette directive se termine de manière traditionnelle par un certain nombre de dispositions relatives aux adaptations futures éventuelles, à la procédure qui devra être suivie ainsi qu'aux abrogations ou dispositions transitoires tout aussi traditionnelles dans ce type de texte législatif.

\section{- 3.7 Les annexes}

Il a déjà été largement question des annexes lors de la présentation générale de la directive. Ces annexes permettent, en effet, de définir des éléments techniques susceptibles d'évoluer avec le progrès technique ou l'expérience. Il est nécessaire d'insister sur l'importance de ces annexes.

En particulier, l'annexe V est essentielle car elle fournit de manière très détaillée ( 51 pages ! !) les bases techniques permettant de définir la qualité écologique, ainsi que les milieux artificiels et fortement modifiés. Elle précise également le dispositif de surveillance et de contrôle du milieu naturel qui devra être mis en place. Malgré l'importance des détails de cette annexe, il n'en reste pas moins que beaucoup de travaux à caractère technique devront être développés dans les tout prochains mois. La Commission Européenne a, pour sa part, pris l'initiative de tels travaux dans trois domaines particuliers : les états de référence pour la qualité écologique, les milieux fortement modifiés et les eaux souterraines.

\section{LA DIRECTIVE-CADRE ET LES EAUX SOUTERRAINES}

\subsection{L'importance des eaux souterraines}

La directive cadre ne pouvait ignorer les eaux souterraines et ceci pour plusieurs raisons. D'abord, ainsi que cela l'a déjà été indiqué, le premier objectif poursuivi consiste à assurer un approvisionnement en eau potable des populations. Or, dans beaucoup de politiques nationales, l'eau souterraine doit servir prioritairement à cet approvisionnement en eau potable des populations. Par ailleurs, au cours de la dernière décennie, une attention toute particulière avait été apportée par les instances communautaires à l'eau souterraine ; un séminaire ministériel sur les eaux souterraines tenu à la Haye en 1991 appelait à lancer un programme d'action visant à la gestion écologiquement viable et à la protection des ressources en eau douce. En 1995, le Conseil des ministres de l'Union Européenne avait réitéré cette demande en souhaitant l'inscrire dans le cadre d'une politique globale de protection des eaux douces.

La protection des ressources en eau souterraine et la recherche d'un bon état durable de ces eaux est donc apparue naturellement comme un volet majeur de la directive cadre. Néanmoins, l'importance des enjeux notamment financiers induits par cet objectif a conduit le Parlement et le Conseil à s'opposer sur les modalités d'atteinte de cet objectif et, en particulier, le délai octroyé aux Etats-membres pour atteindre un bon état des eaux souterraines ainsi que les possibilités de dérogation éventuelles.

\subsection{L'objectif spécifique pour les eaux souterraines}

L'objectif général est énoncé à l'article $1^{\text {er }}$ : les eaux souterraines sont expressément citées parmi les eaux dont il convient d'assurer la protection. Il est, par ailleurs, rappelé qu'il convient d'" assurer un approvisionnement suffisant en eau de surface et en eau souterraine de bonne qualité pour 
les besoins d'une utilisation durable équilibrée et équitable de l'eau ". Comme cela l'a été indiqué, un objectif supplémentaire important concernant la réduction de la pollution anthropogénique des eaux souterraines a été introduit au cours des discussions entre le Conseil et le Parlement.

L'article 2 présente une série de définitions particulières relatives aux eaux souterraines. Par exemple, la définition du bon état qui englobe l'état quantitatif et chimique de l'eau : les normes de qualité prévues dans toutes les dispositions législatives communautaires pertinentes permettent de fixer le niveau du bon état chimique tandis que l'état quantitatif dépend du degré d'incidence des captages sur la ressource. Une notion de ressource disponible d'eau souterraine visant à mieux gérer l'aspect quantitatif a également été introduite

L'article 3 précise dans quel cadre spatial doit se situer l'action à mettre en place, c'est-à-dire le district hydrographique. Apparaît ici une innovation par rapport à la pratique française actuelle dans la mesure où "lorsque les eaux souterraines ne correspondent pas à un bassin hydrographique particulier, elles sont identifiées et intégrées au district hydrographique le plus proche ou le plus approprié ». Il apparaît donc que les nappes souterraines étendues doivent être appréhendées dans leur totalité et "affectées " à un bassin hydrographique particulier; ceci aura des conséquences non négligeables sur les programmes de mesures et les plans de gestion et en particulier sur le périmètre couvert par ces derniers.

L'objectif général de l'article $1^{\text {er }}$ est développé à l'article 4 - et plus particulièrement à l'article 4.1.b) - où il est demandé de prévenir toute détérioration future de l'état des eaux, de restaurer les eaux souterraines polluées afin de parvenir à un bon état de celles-ci dans un délai de seize ans (souhait du Conseil) ou de dix ans (position du Parlement). Il est également prévu d'assurer un équilibre entre les captages et le renouvellement. De plus le Parlement souhaite limiter de manière drastique toute pollution d'origine ponctuelle ou diffuse et demande de développer des actions dès lors qu'il y a une hausse de la concentration en polluants et que cette concentration dépasse la moitié de la valeur-limite spécifiée dans les diverses réglementations.

Dans ce même article 4, des dérogations sont prévues mais elles doivent être motivées par des raisons particulièrement sérieuses telles que l'importance des pressions humaines ainsi que le coût disproportionné des actions nécessaires.

\subsection{L'état des lieux}

La caractérisation initiale des eaux prévue à l'article 5 est, de fait, particulièrement développée à l'annexe II en particulier dans le paragraphe 2 qui précise méticuleusement les méthodologies de classification des eaux souterraines, en tenant compte des pressions humaines et des conditions naturelles existantes. Cette classification doit permettre en particulier de différencier les objectifs à atteindre et de servir de base à des objectifs dérogatoires éventuels. Les articles 6 et 7 n'apportent pas de précisions particulières pour les eaux souterraines en matière de registre des zones protégées ou de conditions de captage.

\subsection{Les instruments nécessaires}

L'article 8 relatif à la surveillance du milieu naturel pose le cadre d'une "surveillance de l'état chimique et quantitatif » des eaux souterraines et renvoie de fait aux dispositions de l'annexe $\mathrm{V}$ et plus particulièrement de son paragraphe 2 qui indique en une dizaine de pages l'ensemble du réseau de surveillance à établir. Il est notamment précisé comment suivre l'état quantitatif et chimique des eaux souterraines, la densité et la fréquence de la surveillance ainsi que l'interprétation qui doit en être faite.
Les articles 9 (tarification incitative) et 10 (approche combinée) n'apportent pas de conditions particulières faites aux eaux souterraines.

\subsection{La programmation}

L'article 11 relatif aux programmes de mesures apporte deux éléments particuliers aux eaux souterraines. Tout d'abord, il est prévu «l'interdiction du rejet direct de polluants dans les eaux souterraines » sauf dans certains cas particuliers relatifs à l'injection de gaz à des fins de stockage, la construction ou le génie civil, ou nécessitées par des fins géothermiques. Par ailleurs, le Parlement souhaite introduire une obligation d'autorisation préalable pour la recharge artificielle des nappes. L'institution d'une autorisation préalable pour les captages d'eau (superficielles et souterraines) importants et susceptible d'avoir un impact sur la qualité des eaux est également prévu.

\subsection{La planification et les stratégies}

Aucune disposition particulière relative aux eaux souterraines n'apparaît dans les articles 13 à 16 qui traitent de ces questions. Les eaux souterraines ne font donc pas l'objet d'une attention particulière dans l'ensemble du dispositif de planification et en particulier la consultation du public n'enjoint pas de tenir compte d'un caractère propre aux eaux souterraines.

\section{- 4.7 Points complémentaires}

Il doit être relevé que dans l'ensemble des directives qui seront abrogées ou reprises au sein de la directive cadre, il est prévu que la directive 80/68/CEE concernant la protection des eaux souterraines contre la pollution causée par certaines substances dangereuses sera abrogée d'ici treize ans. Cela ne signifie cependant pas que les dispositions prévues par cette directive disparaissent puisque l'ensemble de celles-ci sont reprises dans la directive cadre et progressivement développées ; en particulier, la liste des substances dites prioritaires sera élargie conformément à la procédure instaurée par l'article 16 relatif aux stratégies et elle produira ses effets particuliers sur la protection des nappes souterraines.

\section{$\mathrm{V}$ CONCLUSIONS}

La directive cadre pour la protection des ressources en eau s'est efforcée de tenir compte des particularités relatives à une bonne gestion des eaux souterraines, notamment en introduisant la notion spécifique d'état quantitatif qui n'apparaît pas pour les autres compartiments de la ressource en eau. Le niveau de protection des eaux souterraines a fait l'objet d'attentions toutes particulières du Parlement Européen qui a souhaité introduire des dispositions très (trop ?) rigoureuses afin d'améliorer dans les plus brefs délais la qualité de cette ressource. Il convient d'avoir conscience de la difficulté des objectifs à atteindre que fixe la directive cadre dans ce secteur particulier. Les études particulières et spécifiques à réaliser au sein de chaque district permettront de mieux évaluer l'effort important qu'il conviendra de mener afin d'atteindre ces objectifs. De même, l'étude lancée par la Commission devrait permettre de mieux définir les caractéristiques détaillées des réseaux de surveillance et des méthodologies d'évaluation associées.

Cela n'est donc que la première étape d'un programme qui s'étalera sur les années à venir et dont les résultats concrets ne devraient guère être atteints avant la fin de cette décennie. 